\title{
Quantitative stability of fully random mixed-integer two-stage stochastic programs
}

\author{
W. Römisch, S. Vigerske \\ Institute of Mathematics, Humboldt-University Berlin, D-10099 Berlin, Germany
}

The date of receipt and acceptance will be inserted by the editor

\begin{abstract}
Mixed-integer two-stage stochastic programs with fixed recourse matrix, random recourse costs, technology matrix, and right-hand sides are considered. Quantitative continuity properties of its optimal value and solution set are derived when the underlying probability distribution is perturbed with respect to an appropriate probability metric.
\end{abstract}

Key words Stochastic programming - two-stage - mixed-integer - stability - weak convergence - probability metric - discrepancy

\section{Introduction}

Mixed-integer two-stage stochastic programs model a variety of practical decision problems under stochastic uncertainty, e.g., in chemical engineering, power production, and trading planning $[8,13,14]$. The probability distribution of the stochastic programming model reflects the available knowledge on the randomness at the modeling stage. When solving such stochastic programming models, the probability distribution is approximately replaced in most cases by a discrete probability measure with finite support. Hence, perturbing or approximating the probability distribution of such models is an important issue for modeling, theory, and numerical methods in stochastic integer programming. While much is known on the structure and algorithms of/for mixed-integer two-stage stochastic programs (cf. the surveys [11,12, $21,22]$ ), the available (quantitative) stability or statistical estimation results do not cover situations with stochastic costs (or prices) (cf. $[7,18,19])$.

Mixed-integer two-stage stochastic programs are of the form

$$
\min \left\{\int_{\Xi} f_{0}(x, \xi) d P(\xi): x \in X\right\}
$$


where the (first-stage) feasible set $X \subseteq \mathbb{R}^{m}$ is closed, $\Xi$ is a closed subset of $\mathbb{R}^{s}$, the function $f_{0}$ from $\mathbb{R}^{m} \times \Xi$ to the extended reals $\overline{\mathbb{R}}$ is a random lower semicontinuous function, and $P$ belongs to the set of all Borel probability measures $\mathcal{P}(\Xi)$ on $\Xi$. Recall that $f_{0}$ is a random lower semicontinuous function if its epigraphical mapping $\xi \mapsto$ epi $f_{0}(\cdot, \xi):=\left\{(x, r) \in \mathbb{R}^{m} \times \mathbb{R}\right.$ : $\left.f_{0}(x, \xi) \leq r\right\}$ is closed-valued and measurable. In mixed-integer two-stage stochastic programs, $f_{0}$ is of the form

$$
f_{0}(x, \xi)=\langle c, x\rangle+\Phi(q(\xi), h(\xi)-T(\xi) x) \quad\left((x, \xi) \in \mathbb{R}^{m} \times \Xi\right),
$$

where $\Phi(u, t)$ denotes the optimal value of the (second-stage) mixed-integer program (with cost $u$ and right-hand side $t$ ), and $q(\xi), T(\xi)$, and $h(\xi)$ are the stochastic cost, technology matrix, and right-hand side, respectively.

With $v(P)$ and $S(P)$ denoting the optimal value and solution set of (1), respectively, the quantitative stability results for stochastic programs developed in [18] (see [18, Theorems 5 and 9]) imply, in particular, the estimates

$$
\begin{aligned}
& |v(P)-v(Q)| \leq L \sup _{x \in X}\left|\int_{\Xi} f_{0}(x, \xi)(P-Q)(d \xi)\right| \\
& \emptyset \neq S(Q) \subseteq S(P)+\Psi_{P}\left(L \sup _{x \in X}\left|\int_{\Xi} f_{0}(x, \xi)(P-Q)(d \xi)\right|\right),
\end{aligned}
$$

where $L>0$ is some constant, $X$ is assumed to be compact, $\Psi_{P}$ is the conditioning function, and $P$ and $Q$ belong to a suitable subset of $\mathcal{P}(\Xi)$. The function $\Psi_{P}$ depends on the growth behavior of the objective function near the solution set and is specified in (11) of Section 3.

The aim of this paper is to extend the quantitative continuity properties of $v(\cdot)$ and $S(\cdot)$ in $[16,20]$ to cover situations with stochastic costs. To this end, we need quantitative continuity and growth properties of optimal value functions and solution sets of parametric mixed-integer linear programs. Such properties are known for parametric right-hand sides $[4,5,20]$ and parametric costs separately $[1,2,6]$. Since to our knowledge simultaneous perturbation results with respect to right-hand sides and costs are less familiar, we discuss such properties of optimal value functions in Proposition 1. These results are then used in Section 3 to obtain the desired quantitative stability result (Theorem 1) for fully random mixed-integer two-stage stochastic programs with fixed recourse. The relevant probability metric (9) on subsets of $\mathcal{P}(\Xi)$ and its relations to Fortet-Mourier metrics and polyhedral discrepancies are also discussed (Remark 2). The latter metrics may be used for designing moderately sized discrete approximations to $P$ by optimal scenario reduction of discrete probability measures $[9,10]$.

\section{Infima of mixed-integer linear programs}

Consider the parametric mixed-integer linear program

$$
\min \left\{\left\langle c_{x}, x\right\rangle+\left\langle c_{y}, y\right\rangle: A_{x} x+A_{y} y \leq b, x \in \mathbb{Z}^{n}, y \in \mathbb{R}^{m-n}\right\}
$$


with $c=\left(c_{x}, c_{y}\right) \in \mathbb{R}^{m}$ and $b \in \mathbb{R}^{r}$ playing the role of the parameters and $A=\left(A_{x}, A_{y}\right) \in \mathbb{Q}^{r \times m}$. Let $M(b), v(b, c)$, and $S(b, c)$ denote the feasible set, optimal value, and solution set of (5), respectively, i.e.,

$$
\begin{aligned}
M(b) & :=\left\{(x, y) \in \mathbb{Z}^{n} \times \mathbb{R}^{m-n}: A(x, y) \leq b\right\} \\
v(b, c) & :=\inf \{\langle c,(x, y)\rangle:(x, y) \in M(b)\} \\
S(b, c) & :=\{(x, y) \in M(b):\langle c,(x, y)\rangle=v(b, c)\} .
\end{aligned}
$$

Let $\mathcal{K}$ denote the polyhedral cone $\left\{(x, y) \in \mathbb{R}^{m}: A_{x} x+A_{y} y \leq 0\right\}$ and $\mathcal{K}^{*}$ its polar cone. Observe that $v(b, c)$ is finite for $b \in \mathcal{B}:=\operatorname{dom} M$ and $c \in-\mathcal{K}^{*}$. Further, denote by $\operatorname{Pr}_{x} M(b)$ the projection of $M(b)$ onto the $x$-space, and

$$
\mathcal{B}^{*}\left(b^{0}\right):=\left\{b \in \mathcal{B}: \operatorname{Pr}_{x} M(b)=\operatorname{Pr}_{x} M\left(b^{0}\right)\right\} \quad\left(b^{0} \in \mathcal{B}\right)
$$

be the set of right-hand sides on which the projection of $M(b)$ onto the $x$ space is constant. It is well known (see [1, Chapter 5.6]) that the sets $\mathcal{B}^{*}\left(b^{0}\right)$ are continuity regions of the function $b \mapsto v(b, c)$. These regions are further characterized by the following result (Lemma 5.6.1 and 5.6.2 in [1]).

Lemma $1 \mathcal{B}$ is a connected set equal to the union of a countable family of convex polyhedral cones each of which is obtained by a translation of the $r$-dimensional cone $T:=\left\{t \in \mathbb{R}^{r}: \exists y \in \mathbb{R}^{m-n}\right.$ such that $\left.t \geq A_{y} y\right\}$.

For each $b^{0} \in \mathcal{B}$, there exists $t^{0} \in \mathcal{B}$ and a finite set $N \subseteq \mathbb{Z}^{n} \backslash \operatorname{Pr}_{x} M\left(b^{0}\right)$ such that

$$
\mathcal{B}^{*}\left(b^{0}\right)=\left(t^{0}+T\right) \backslash \bigcap_{z \in N}\left(A_{x} z+T\right) .
$$

If $\operatorname{Pr}_{x} M\left(b^{0}\right)=\mathbb{Z}^{n}$, then $N=\emptyset$ and $\mathcal{B}^{*}\left(b^{0}\right)=t^{0}+T$ for some $t^{0} \in \mathcal{B}$.

In the following we assume that the projection $\operatorname{Pr}_{x} S(b, c)$ of the solution set intersects with a ball of some radius $K$ for all $(b, c) \in \mathcal{B} \times-\mathcal{K}^{*}$. This allows us to extend Lemma 2.3 in [20] and show local Lipschitz-continuity of the optimal value of (5) with respect to simultaneous perturbations of the right-hand side and the objective function coefficients where the right-hand side perturbation does not leave the continuity region $B^{*}(b)$. Otherwise, for arbitrary right-hand sides, a quasi-Lipschitz property of the value function of (5) can be shown. For the proof of our next result we refer to the appendix.

Proposition 1 (i) Let $b \in \mathcal{B}, b^{\prime} \in \mathcal{B}^{*}(b)$, and $c, c^{\prime} \in-\mathcal{K}^{*}$. Assume that for some constant $K \geq 1, \operatorname{Pr}_{x} S\left(b^{\prime}, c\right) \cap \mathbb{B}(0, K) \neq \emptyset$ and $\operatorname{Pr}_{x} S\left(b^{\prime}, c^{\prime}\right) \cap \mathbb{B}(0, K) \neq$ $\emptyset$. Then the estimate

$$
\left|v(b, c)-v\left(b^{\prime}, c^{\prime}\right)\right| \leq L_{1} \max \left\{\|c\|,\left\|c^{\prime}\right\|\right\}\left\|b-b^{\prime}\right\|+L_{2} \max \left\{\|b\|,\left\|b^{\prime}\right\|, K\right\}\left\|c-c^{\prime}\right\|
$$

holds, where the constants $L_{1}$ and $L_{2}$ depend on $A$ only.

(ii) Let $b, b^{\prime} \in \mathcal{B}$ and $c, c^{\prime} \in-\mathcal{K}^{*}$. Then we have

$\left|v(b, c)-v\left(b^{\prime}, c^{\prime}\right)\right| \leq \max \left\{\|c\|,\left\|c^{\prime}\right\|\right\}\left(\tilde{L}\left\|b-b^{\prime}\right\|+\ell\right)+\tilde{L} \max \left\{\|b\|,\left\|b^{\prime}\right\|\right\}\left\|c-c^{\prime}\right\|$, where the constants $\tilde{L}$ and $\ell$ depend on $A$ only. 
Remark 1 Observe that for mixed-integer linear programs with bounded integer variables (e.g., mixed-binary programs), the integer part of the feasible points is uniformly bounded. This bound yields a suitable constant $K$.

The following result is [4, Theorem 2.1] and can be found in similar form also in [2]. Together with Proposition 1 it is needed to prove Lemma 3.

Lemma 2 Let $c \in-\mathcal{K}^{*}$. The mapping $b \mapsto S(b, c)$ is quasi-Lipschitz continuous on $\mathcal{B}$ with constants $\bar{L}_{1}$ and $\bar{L}_{2}$ not depending on $b$ and $c$, i.e.,

$$
d_{H}\left(S(b, c), S\left(b^{\prime}, c\right)\right) \leq \bar{L}_{1}\left\|b-b^{\prime}\right\|+\bar{L}_{2},
$$

where $d_{H}$ denotes the Hausdorff distance on subsets of $\mathbb{R}^{m}$.

\section{Quantitative stability of mixed-integer two-stage stochastic programs}

Let us consider the stochastic program

$$
\min \left\{\langle c, x\rangle+\int_{\Xi} \Phi(q(\xi), h(\xi)-T(\xi) x) P(d \xi): x \in X\right\},
$$

where $\Phi$ is the infimum function of a mixed-integer linear program given by

$$
\Phi(u, t):=\inf \left\{\left\langle u_{1}, y\right\rangle+\left\langle u_{2}, \bar{y}\right\rangle: W y+\bar{W} \bar{y} \leq t, y \in \mathbb{Z}^{\hat{m}}, \bar{y} \in \mathbb{R}^{\bar{m}}\right\}
$$

for all pairs $(u, t) \in \mathbb{R}^{\hat{m}+\bar{m}} \times \mathbb{R}^{r}$, and $c \in \mathbb{R}^{m}, X$ is a closed subset of $\mathbb{R}^{m}, \Xi$ a polyhedron in $\mathbb{R}^{s}, W$ and $\bar{W}$ are $(r, \hat{m})$ - and $(r, \bar{m})$-matrices, respectively, $q(\xi) \in \mathbb{R}^{\hat{m}+\bar{m}}, h(\xi) \in \mathbb{R}^{r}$, and the $(r, m)$-matrix $T(\xi)$ are affine functions of $\xi \in \mathbb{R}^{s}$, and $P \in \mathcal{P}(\Xi)$.

We need the following conditions to have the model (6) well-defined:

(B1) The matrices $W$ and $\bar{W}$ have only rational elements.

(B2) For each pair $(x, \xi) \in X \times \Xi$ it holds that $h(\xi)-T(\xi) x \in \mathcal{T}$, where

$$
\mathcal{T}:=\left\{t \in \mathbb{R}^{r}: \exists(y, \bar{y}) \in \mathbb{Z}^{\hat{m}} \times \mathbb{R}^{\bar{m}} \text { such that } W y+\bar{W} \bar{y} \leq t\right\} .
$$

(B3) For each $\xi \in \Xi$ the recourse cost $q(\xi)$ belongs to the dual feasible set $\mathcal{U}:=\left\{u=\left(u_{1}, u_{2}\right) \in \mathbb{R}^{\hat{m}+\bar{m}}: \exists z \in \mathbb{R}_{-}^{r}\right.$ such that $\left.W^{\top} z=u_{1}, \bar{W}^{\top} z=u_{2}\right\}$.

(B4) The integer part of all second-stage feasible solutions is uniformly bounded for all $t=h(\xi)-T(\xi) x,(x, \xi) \in X \times \Xi$ (e.g., in case of mixedbinary programs).

(B5) $P \in \mathcal{P}_{2}(\Xi)$, i.e., $P \in \mathcal{P}(\Xi)$ and $\int_{\Xi}\|\xi\|^{2} P(d \xi)<+\infty$.

The conditions (B2) and (B3) mean relatively complete recourse and dual feasibility, respectively. We note that (B2) and (B3) imply $\Phi(u, t)$ to be finite for all $(u, t) \in \mathcal{U} \times \mathcal{T}$. The following additional properties of the value function $\Phi$ on $\mathcal{U} \times \mathcal{T}$ are important in the context of this paper. 
Lemma 3 Assume (B1)-(B4). Then there exists a countable partition of $\mathcal{T}$ into Borel subsets $\mathcal{B}_{i}$, i.e., $\mathcal{T}=\bigcup_{i \in \mathbb{N}} \mathcal{B}_{i}$ such that

(i) $\mathcal{B}_{i}=\left(b_{i}+T\right) \backslash \bigcup_{j=1}^{N_{0}}\left(b_{i, j}+T\right)$, where $b_{i}, b_{i, j} \in \mathbb{R}^{r}, i \in \mathbb{N}, j=1, \ldots, N_{0}$, $N_{0} \in \mathbb{N}$ does not depend on $i$, and $T:=\left\{t \in \mathbb{R}^{r}: \exists y \geq 0\right.$ such that $t \geq$ $W y\}$. Moreover there exists an $N_{1} \in \mathbb{N}$ such that for all $t \in \mathcal{T}$ the ball $\mathbb{B}(t, 1)$ in $\mathbb{R}^{r}$ is intersected by at most $N_{1}$ different subsets $\mathcal{B}_{i}$.

(ii) the restriction $\left.\Phi\right|_{\mathcal{U} \times \mathcal{B}_{i}^{\prime}}$, where $\mathcal{B}_{i}^{\prime}:=\mathcal{B}_{i} \cap\{h(\xi)-T(\xi) x \mid(x, \xi) \in X \times \Xi\}$, has the property that there exists a constant $L>0$ independent of $i$, s.t.

$$
|\Phi(u, t)-\Phi(\tilde{u}, \tilde{t})| \leq L(\max \{1,\|t\|,\|\tilde{t}\|\}\|u-\tilde{u}\|+\max \{1,\|u\|,\|\tilde{u}\|\}\|t-\tilde{t}\|) .
$$

Furthermore, the function $\Phi$ is lower semicontinuous and piecewise polyhedral on $\mathcal{U} \times \mathcal{T}$ and there exist constants $D, d>0$ such that it holds for all pairs $(u, t),(\tilde{u}, \tilde{t}) \in \mathcal{U} \times \mathcal{T}$ :

$|\Phi(u, t)-\Phi(\tilde{u}, \tilde{t})| \leq D(\max \{1,\|t\|,\|\tilde{t}\|\}(\|u-\tilde{u}\|+d)+\max \{1,\|u\|,\|\tilde{u}\|\}\|t-\tilde{t}\|)$.

The first part of (i) is Lemma 1 . The second part is an extension of [20, Lemma 2.5] to the function $\Phi(u, t)$ since the relevant constants in its proof do not depend on the objective function as recalled in Lemma 2. Part (ii) and the quasi-Lipschitz property of $\Phi$ is Proposition 1.

The representation of $\Phi$ is given on countably many (possibly unbounded) Borel sets. This requires to incorporate the tail behavior of $P$ and leads to the following representation of the function $f_{0}$.

Proposition 2 Assume (B1)-(B4) and $X$ be bounded. For each $R \geq 1$ and $x \in X$ there exist disjoint Borel subsets $\Xi_{j, x}^{R}$ of $\Xi, j=1, \ldots, \nu$, whose closures are polyhedra with a uniformly bounded number of faces such that

$$
f_{0}(x, \xi)=\sum_{j=0}^{\nu}(\langle c, x\rangle+\Phi(q(\xi), h(\xi)-T(\xi) x)) \mathbb{1}_{\Xi_{j, x}^{R}}(\xi) \quad((x, \xi) \in X \times \Xi)
$$

is Lipschitz continuous with respect to $\xi$ on each $\Xi_{j, x}^{R}, j=1, \ldots, \nu$, with some uniform Lipschitz constant. Here, $\Xi_{0, x}^{R}:=\Xi \backslash \cup_{j=1}^{\nu} \Xi_{j, x}^{R}$ is contained in $\left\{\xi \in \mathbb{R}^{s}:\|\xi\|_{\infty}>R\right\}, \nu$ is bounded by a multiple of $R^{r}$ and $\mathbb{1}_{A}$ denotes the characteristic function of a set $A$.

Proof Since $q(\cdot), h(\cdot)$ and $T(\cdot)$ are affine linear functions and $X$ is bounded, there exists a constant $C>0$ such that the estimate

$$
\max \left\{\|q(\xi)\|_{\infty},\|h(\xi)-T(\xi) x\|_{\infty}\right\} \leq C \max \left\{1,\|\xi\|_{\infty}\right\}
$$

holds for each pair in $X \times \Xi$. Let $R \geq 1$ and $\mathcal{T}_{R}:=\mathcal{T} \cap C R \mathbb{B}_{\infty}$, where $\mathbb{B}_{\infty}$ is the unit ball w.r.t. the maximum norm $\|\cdot\|_{\infty}$. As in [18, Proposition 34] there exist a number $\nu \in \mathbb{N}$ and disjoint Borel subsets $\left\{B_{j}\right\}_{j=1}^{\nu}$ of $C R \mathbb{B}_{\infty}$ such that their closures are polyhedra and their union contains $\mathcal{T}_{R}$. Furthermore, when arguing as in the proof of [20, Proposition 3.1], $\nu$ is bounded above 
by $\kappa R^{r}$, where the constant $\kappa>0$ is independent of $R$. Now, let $x \in X$ and consider the following disjoint Borel subsets of $\Xi$ :

$$
\begin{aligned}
& \Xi_{j, x}^{R}:=\left\{\xi \in \Xi: h(\xi)-T(\xi) x \in B_{j},\|\xi\|_{\infty} \leq R\right\} \quad(j=1, \ldots, \nu), \\
& \Xi_{0, x}^{R}:=\Xi \backslash \bigcup_{j=1}^{\nu} \Xi_{j, x}^{R} \subseteq\left\{\xi \in \Xi:\|\xi\|_{\infty}>R\right\} .
\end{aligned}
$$

Let $x \in X$ and $\xi, \xi^{\prime} \in \Xi_{j, x}^{R}$ for some $j \in\{1, \ldots, \nu\}$. By Lemma 3 we obtain

$$
\begin{aligned}
\left|f_{0}(x, \xi)-f_{0}\left(x, \xi^{\prime}\right)\right|= & \left|\Phi(q(\xi), h(\xi)-T(\xi) x)-\Phi\left(q\left(\xi^{\prime}\right), h\left(\xi^{\prime}\right)-T\left(\xi^{\prime}\right) x\right)\right| \\
\leq & L\left(\operatorname { m a x } \{ 1 , \| q ( \xi ) \| _ { \infty } , \| q ( \xi ^ { \prime } ) \| _ { \infty } \} \left(\left\|h(\xi)-h\left(\xi^{\prime}\right)\right\|_{\infty}\right.\right. \\
& \left.+\left\|\left(T(\xi)-T\left(\xi^{\prime}\right)\right) x\right\|_{\infty}\right)+\max \left\{1,\|h(\xi)-T(\xi) x\|_{\infty},\right. \\
& \left.\left.\left\|h\left(\xi^{\prime}\right)-T\left(\xi^{\prime}\right) x\right\|_{\infty}\right\}\left\|q(\xi)-q\left(\xi^{\prime}\right)\right\|_{\infty}\right) \\
\leq & L C R\left(\left\|h(\xi)-h\left(\xi^{\prime}\right)\right\|_{\infty}+\left\|\left(T(\xi)-T\left(\xi^{\prime}\right)\right) x\right\|_{\infty}\right. \\
& \left.+\left\|q(\xi)-q\left(\xi^{\prime}\right)\right\|_{\infty}\right) \\
\leq & L_{1} R\left\|\xi-\xi^{\prime}\right\|_{\infty},
\end{aligned}
$$

where we used (8) for $\xi, \xi^{\prime} \in \Xi_{j, x}^{R}$, affine linearity of $q(\cdot), h(\cdot)$, and $T(\cdot)$, and boundedness of $X$. We note that the constant $L_{1}$ is independ of $R$.

In order to state quantitative stability results for model (6) and inspired by the estimates (3) and (4), we need a distance of probability measures that captures the behavior of $f_{0}(x, \cdot)(x \in X)$ in its continuity regions and the shape of these regions, respectively. This leads us to the following probability metric on $\mathcal{P}_{2}(\Xi)$ for some $k \in \mathbb{N}$ :

$$
\zeta_{2, \mathrm{ph}_{k}}(P, Q):=\sup \left\{\left|\int_{B} f(\xi)(P-Q)(d \xi)\right|: f \in \mathcal{F}_{2}(\Xi), B \in \mathcal{B}_{\mathrm{ph}_{k}}(\Xi)\right\} .
$$

Here, $\mathcal{B}_{\mathrm{ph}_{k}}(\Xi)$ denotes the set of all polyhedra being subsets of $\Xi$ and having at most $k$ faces. The set $\mathcal{F}_{2}(\Xi)$ contains all functions $f: \Xi \rightarrow \mathbb{R}$ such that

$$
|f(\xi)| \leq \max \left\{1,\|\xi\|^{2}\right\} \text { and }|f(\xi)-f(\tilde{\xi})| \leq \max \{1,\|\xi\|,\|\tilde{\xi}\|\}\|\xi-\tilde{\xi}\|
$$

holds for all $\xi, \tilde{\xi} \in \Xi$. We note that, unfortunately, the growth condition on $f$ is missing in the description of the set of functions in $[16,18]$.

Before stating the main result, we define the function $\phi_{P}$ on $\mathbb{R}_{+}$characterizing the tail behavior of $P$ by $\phi_{P}(0)=0$ and

$$
\phi_{P}(t):=\inf _{R \geq 1}\left\{R^{r+1} t+\int_{\left\{\xi \in \Xi:\|\xi\|_{\infty}>R\right\}}\|\xi\|_{\infty}^{2} P(d \xi)\right\} \quad(t>0),
$$

and the conditioning function $\Psi_{P}$ by

$$
\Psi_{P}(\eta):=\eta+\psi_{P}^{-1}(2 \eta) \quad\left(\eta \in \mathbb{R}_{+}\right),
$$

where the growth function $\psi_{P}$ on $\mathbb{R}_{+}$is

$$
\psi_{P}(\tau):=\min \left\{\int_{\Xi} f_{0}(x, \xi) P(d \xi)-v(P): d(x, S(P)) \geq \tau, x \in X\right\}
$$


with inverse $\psi_{P}^{-1}(t):=\sup \left\{\tau \in \mathbb{R}_{+}: \psi_{P}(\tau) \leq t\right\}$. The functions $\phi_{P}$ and $\psi_{P}$ are nondecreasing, $\Psi_{P}$ is increasing and all functions vanish at 0 . Furthermore, one has $\psi_{P}(\tau)>0$ if $\tau>0$ and $\Psi_{P}(\eta) \searrow 0$ if $\eta \searrow 0$.

Theorem 1 Let the conditions (B1)-(B5) be satisfied and $X$ be compact.

Then there exist constants $L>0$ and $k \in \mathbb{N}$ such that

$$
\begin{aligned}
|v(P)-v(Q)| & \leq L \phi_{P}\left(\zeta_{2, \mathrm{ph}_{k}}(P, Q)\right) \\
\emptyset \neq S(Q) & \subseteq S(P)+\Psi_{P}\left(L \phi_{P}\left(\zeta_{2, \mathrm{ph}_{k}}(P, Q)\right)\right) \mathbb{B},
\end{aligned}
$$

for each $Q \in \mathcal{P}_{2}(\Xi)$. If $\int_{\Xi}\|\xi\|^{p} P(d \xi)<+\infty$ for some $p>2$, the estimate $\phi_{P}(t) \leq C t^{\frac{p-2}{p+r-1}}$ holds for every $t \geq 0$ and some constant $C>0$.

Proof Since the function $\Phi$ is lower semicontinuous on $\mathcal{U} \times \mathcal{T}$ (Lemma 3 ), $f_{0}$ is lower semicontinuous on $X \times \Xi$ and, hence, a random lower semicontinuous function [17, Example 14.31]. Using Lemma 3 we obtain the estimate

$$
\begin{aligned}
\left|f_{0}(x, \xi)\right| \leq & \|c\|\|x\|+D[\max \{1,\|h(\xi)\|+\|T(\xi)\|\|x\|\}(\|q(\xi)\|+d) \\
& +\max \{1,\|q(\xi)\|\}(\|h(\xi)\|+\|T(\xi)\|\|x\|)] \\
\leq & C_{1} \max \left\{1,\|\xi\|^{2}\right\}
\end{aligned}
$$

for each pair $(x, \xi) \in X \times \Xi$ and some constant $C_{1}$. Hence, the objective function $\langle c, x\rangle+\int_{\Xi} \Phi(q(\xi), h(\xi)-T(\xi) x) Q(d \xi)$ is finite (if $Q \in \mathcal{P}_{2}(\Xi)$ ) and lower semicontinuous (due to Fatou's lemma). Since $X$ is compact, the solution set $S(Q)$ is nonempty.

From Proposition 2 we know that, for each $R \geq 1$ and $x \in X$, there exist Borel subsets $\Xi_{j, x}^{R}, j=1, \ldots, \nu$, of $\Xi$ such that the function $f_{j, x}^{R}(\cdot):=$ $f_{0}(x, \cdot) \mathbb{1}_{\Xi_{j, x}^{R}}$ is Lipschitz continuous on $\Xi_{j, x}^{R}$ with constant $L_{1} R$. We extend each function $f_{j, x}^{R}(\cdot)$ to the whole of $\Xi$ by preserving the Lipschitz constant. Then we have $\frac{1}{L_{1} R} f_{j, x}^{R}(\cdot) \in \mathcal{F}_{2}(\Xi)$. Furthermore, Proposition 2 implies that the closures of $\Xi_{j, x}^{R}$ are contained in $\mathcal{B}_{\mathrm{ph}_{k}}(\Xi)$ for some $k \in \mathbb{N}$, that the number $\nu$ is bounded above by $\kappa R^{r}$, where the constant $\kappa>0$ is independent on $R$, and that $\Xi_{0, x}^{R}:=\Xi \backslash \bigcup_{j=1}^{\nu} \Xi_{j, x}^{R}$ is a subset of $\left\{\xi \in \Xi:\|\xi\|_{\infty}>R\right\}$. For each $Q \in \mathcal{P}_{2}(\Xi)$ and $x \in X$ we obtain

$$
\begin{aligned}
& \left|\int_{\Xi} f_{0}(x, \xi)(P-Q)(d \xi)\right|=\left|\sum_{j=0}^{\nu} \int_{\Xi_{j, x}^{R}} f_{0}(x, \xi)(P-Q)(d \xi)\right| \\
& \quad \leq \sum_{j=1}^{\nu}\left|\int_{\Xi_{j, x}^{R}} f_{j, x}^{R}(\xi)(P-Q)(d \xi)\right|+I_{x}^{R}(P, Q) \\
& \leq \nu L_{1} R \sup _{\substack{f \in \mathcal{F}_{2}(\Xi) \\
j=1, \ldots, \nu}}\left|\int_{\Xi} f(\xi) \chi_{\Xi_{j, x}^{R}}(\xi)(P-Q)(d \xi)\right|+I_{x}^{R}(P, Q),
\end{aligned}
$$

where the last summand on the right-hand side is given by

$$
I_{x}^{R}(P, Q):=\left|\int_{\Xi_{0, x}^{R}} f_{0}(x, \xi)(P-Q)(d \xi)\right| .
$$


Using $\nu \leq \kappa R^{r}$ and arguing as in [18, Theorem 35] we continue

$$
\left|\int_{\Xi} f_{0}(x, \xi)(P-Q)(d \xi)\right| \leq \kappa L_{1} R^{r+1} \zeta_{2, \mathrm{ph}_{k}}(P, Q)+I_{x}^{R}(P, Q) .
$$

For the term $I_{x}^{R}(P, Q)$ we use the estimate $\left|f_{0}(x, \xi)\right| \leq C_{1}\|\xi\|^{2}$ for any pair $(x, \xi) \in X \times\left\{\xi \in \Xi:\|\xi\|_{\infty}>R\right\}$ and the norming constant $C_{2}$ such that $\|\xi\| \leq C_{2}\|\xi\|_{\infty}$ holds for all $\xi \in \mathbb{R}^{s}$. We get

$$
I_{x}^{R}(P, Q) \leq C_{1} C_{2}^{2} \int_{\left\{\xi \in \Xi:\|\xi\|_{\infty}>R\right\}}\|\xi\|_{\infty}^{2}(P+Q)(d \xi) .
$$

Since the set $\left\{\xi \in \Xi:\|\xi\|_{\infty}>R\right\}$ can be covered by $2^{s}$ intersections of $\Xi$ with open halfspaces (whose closures belong to $\mathcal{B}_{\mathrm{ph}_{k}}(\Xi)$ ), we can estimate

$$
\int_{\left\{\xi \in \Xi:\|\xi\|_{\infty}>R\right\}}\|\xi\|_{\infty}^{2} Q(d \xi) \leq 2^{s} \zeta_{2, \mathrm{ph}_{k}}(P, Q)+\int_{\left\{\xi \in \Xi:\|\xi\|_{\infty}>R\right\}}\|\xi\|_{\infty}^{2} P(d \xi) .
$$

Hence, combining the last three estimates we get

$$
\begin{aligned}
\sup _{x \in X}\left|\int_{\Xi} f_{0}(x, \xi)(P-Q)(d \xi)\right| \leq & \left(\kappa L_{1} R^{r+1}+C_{1} C_{2}^{2} 2^{s}\right) \zeta_{2, \mathrm{ph}_{k}}(P, Q) \\
& +2 C_{1} C_{2}^{2} \int_{\{\xi \in \Xi:\|\xi\|>R\}}\|\xi\|_{\infty}^{2} P(d \xi)
\end{aligned}
$$

for any $R \geq 1$. Taking the infimum with respect to $R \geq 1$ we obtain

$$
\sup _{x \in X}\left|\int_{\Xi} f_{0}(x, \xi)(P-Q)(d \xi)\right| \leq \hat{C} \phi_{P}\left(\zeta_{2, \operatorname{ph}_{k}}(P, Q)\right)
$$

with some constant $\hat{C}>0$. Now, the result is a consequence of the estimates (3) and (4). If $\int_{\Xi}\|\xi\|^{p} d P(\xi)<+\infty$ for some $p>2$, it holds that $\int_{\left\{\xi \in \Xi:\|\xi\|_{\infty}>R\right\}}\|\xi\|_{\infty}^{2} d P(\xi) \leq R^{2-p} \int_{\Xi}\|\xi\|_{\infty}^{p} P(d \xi)$ by Markov's inequality. The desired estimate follows by inserting $R=t^{-\frac{1}{p+r-1}}$ for small $t>0$ into the function whose infinum w.r.t. $R \geq 1$ is $\phi_{P}(t)$ :

$$
\phi_{P}(t) \leq t^{-\frac{r+1}{p+r-1}+1}+t^{\frac{p-2}{p+r-1}} \int_{\Xi}\|\xi\|_{\infty}^{p} P(d \xi) \leq C t^{\frac{p-2}{p+r-1}}
$$

The boundedness condition on $X$ may be relaxed if localized optimal values and solution sets are considered (see [18]). In case that the underlying distribution $P$ and its perturbations $Q$ have supports in some bounded subset $\Xi$ of $\mathbb{R}^{s}$, the stability result improves slightly.

Corollary 1 Let the conditions (B1)-(B4) be satisfied, $P \in \mathcal{P}(\Xi), X$ and $\Xi$ be bounded. Then there exist constants $L>0$ and $k \in \mathbb{N}$ such that

$$
\begin{aligned}
|v(P)-v(Q)| & \leq L \zeta_{2, \mathrm{ph}_{k}}(P, Q) \\
\emptyset \neq S(Q) & \subseteq S(P)+\Psi_{P}\left(L \zeta_{2, \mathrm{ph}_{k}}(P, Q)\right) \mathbb{B},
\end{aligned}
$$

holds for each $Q \in \mathcal{P}(\Xi)$. 
Proof Since $\Xi$ is bounded, we have $\mathcal{P}_{2}(\Xi)=\mathcal{P}(\Xi)$. Moreover, the function $\phi_{P}(t)$ (see (10)) can be estimated by $R^{r+1} t$ for some sufficiently large $R>0$. Hence, Theorem 1 implies the assertion.

Remark 2 Since $\Xi \in \mathcal{B}_{\mathrm{ph}_{k}}(\Xi)$ for some $k \in \mathbb{N}$, we obtain from (9) by choosing $B:=\Xi$ and $f \equiv 1$, respectively,

$$
\max \left\{\zeta_{2}(P, Q), \alpha_{\mathrm{ph}_{k}}(P, Q)\right\} \leq \zeta_{2, \mathrm{ph}_{k}}(P, Q)
$$

for all $P, Q \in \mathcal{P}_{2}(\Xi)$. Here, $\zeta_{2}$ and $\alpha_{\mathrm{ph}_{k}}$ denote the second order FortetMourier metric [15, Section 5.1] and the polyhedral discrepancy

$$
\begin{aligned}
\zeta_{2}(P, Q) & :=\sup _{f \in \mathcal{F}_{2}(\Xi)}\left|\int_{\Xi} f(\xi) P(d \xi)-\int_{\Xi} f(\xi) Q(d \xi)\right| \\
\alpha_{\mathrm{ph}_{k}}(P, Q) & :=\sup _{B \in \mathcal{B}_{\mathrm{ph}_{k}}(\Xi)}|P(B)-Q(B)|,
\end{aligned}
$$

respectively. Hence, convergence with respect to $\zeta_{2, \mathrm{ph}_{k}}$ implies weak convergence (see [3]), convergence of second order absolute moments, and convergence with respect to the polyhedral discrepancy $\alpha_{\mathrm{ph}_{k}}$. For bounded $\Xi \subset \mathbb{R}^{s}$ the technique in the proof of [20, Proposition 3.1] can be employed to obtain

$$
\zeta_{2, \mathrm{ph}_{k}}(P, Q) \leq C_{s} \alpha_{\mathrm{ph}_{k}}(P, Q)^{\frac{1}{s+1}} \quad(P, Q \in \mathcal{P}(\Xi))
$$

for some constant $C_{s}>0$. In view of (14), (15) the metric $\zeta_{2, \mathrm{ph}_{k}}$ is stronger than $\alpha_{\mathrm{ph}_{k}}$ in general, but in case of bounded $\Xi$ both distances metrize the same topology on $\mathcal{P}(\Xi)$.

For more specific models (6), improvements of the above results may be obtained by exploiting specific recourse structures, i.e., by using additional information on the shape of the sets $\mathcal{B}_{i}$ in Lemma 3 and on the behavior of the (value) function $\Phi$ on these sets. This may lead to stability results with respect to probability metrics that are (much) weaker than $\zeta_{2, \mathrm{ph}_{k}}$. For example, if $q$ and $T$ are fixed and $h(\cdot)$ is of the form $h(\xi):=\xi$ (i.e., $r=s$ ), the closures of the $\mathcal{B}_{i}$ belong to a class of polyhedra which is completely characterized in [20, Section 3]. If, in addition, the model has pure integer recourse, the stability result is valid with respect to the Kolmogorov metric

$$
d_{\mathrm{K}}(P, Q):=\sup _{z \in \mathbb{R}^{s}}|P((-\infty, z])-Q((-\infty, z])|
$$

on $\mathcal{P}(\Xi)$ instead of $\zeta_{2, \mathrm{ph}_{k}}$ if $\Xi$ is bounded (see also [20, Proposition 3.4]).

\section{A Proof of Proposition 1}

Let $b \in \mathcal{B}, b^{\prime} \in \mathcal{B}^{*}(b)$, and $c, c^{\prime} \in-\mathcal{K}^{*}$ be given. To show local Lipschitz continuity of $v(b, c)$, we estimate

$$
\left|v(b, c)-v\left(b^{\prime}, c^{\prime}\right)\right| \leq\left|v(b, c)-v\left(b^{\prime}, c\right)\right|+\left|v\left(b^{\prime}, c\right)-v\left(b^{\prime}, c^{\prime}\right)\right| .
$$


For the first difference we can proceed as for the proof of Lemma 2.3 in [20]. It is repeated here to keep the paper self-contained. We write (5) as

$$
\min \left\{\left\langle c_{x}, x\right\rangle+\Psi\left(c_{y}, b-A_{x} x\right): x \in \operatorname{Pr}_{x} M(b)\right\}
$$

where $\Psi\left(c_{y}, \tilde{b}\right):=\min \left\{\left\langle c_{y}, y\right\rangle: A_{y} y \leq \tilde{b}\right\}$. Since $\Psi\left(c_{y}, \tilde{b}\right)$ is the optimal value function of a linear program and finite for $b \in \mathcal{B}, c^{\prime} \in-\mathcal{K}^{*}$, there exist finitely many matrices $C_{j}$, which depend on $A_{y}$ only, such that $\Psi\left(c_{y}, \tilde{b}\right)=$ $\max _{j}\left\langle\tilde{b}, C_{j} c_{y}\right\rangle$ (cf. [23]). Let $L_{1}:=\max _{j}\left\|C_{j}\right\|$. Then, for $c_{y}$ fixed,

$$
\left|\Psi\left(c_{y}, \tilde{b}\right)-\Psi\left(c_{y}, \tilde{b}^{\prime}\right)\right| \leq L_{1}\left\|c_{y}\right\|\left\|\tilde{b}-\tilde{b}^{\prime}\right\|
$$

Let $(x, y) \in S(b, c),\left(x^{\prime}, y^{\prime}\right) \in S\left(b^{\prime}, c\right)$. Since $\operatorname{Pr}_{x} M(b)=\operatorname{Pr}_{x} M\left(b^{\prime}\right)$, we have

$$
\begin{aligned}
v(b, c)-v\left(b^{\prime}, c\right) & \leq\left\langle c_{x}, x^{\prime}\right\rangle+\Psi\left(c_{y}, b-A_{x} x^{\prime}\right)-\left\langle c_{x}, x^{\prime}\right\rangle-\Psi\left(c_{y}, b^{\prime}-A_{x} x^{\prime}\right) \\
& \leq L_{1}\|c\|\left\|b-b^{\prime}\right\| .
\end{aligned}
$$

Due to symmetry the same estimate holds for $v\left(b^{\prime}, c\right)-v(b, c)$.

To estimate $\left|v\left(b^{\prime}, c\right)-v\left(b^{\prime}, c^{\prime}\right)\right|$ we take $(x, y) \in S\left(b^{\prime}, c\right)$ and $\left(x^{\prime}, y^{\prime}\right) \in$ $S\left(b^{\prime}, c^{\prime}\right)$ such that $x, x^{\prime} \in \mathbb{B}(0, K), y=C_{j}\left(b^{\prime}-A_{x} x\right)$, and $y^{\prime}=C_{j^{\prime}}\left(b^{\prime}-A_{x} x^{\prime}\right)$ for some indices $j$ and $j^{\prime}$. Since $b^{\prime}$ is fixed, it is $v\left(b^{\prime}, c\right) \leq\left\langle c_{x}, x^{\prime}\right\rangle+\left\langle c_{y}, y^{\prime}\right\rangle$ and $v\left(b^{\prime}, c^{\prime}\right) \leq\left\langle c_{x}^{\prime}, x\right\rangle+\left\langle c_{y}^{\prime}, y\right\rangle$. Hence,

$$
\left|v\left(b^{\prime}, c\right)-v\left(b^{\prime}, c^{\prime}\right)\right| \leq \max \left\{\|(x, y)\|,\left\|\left(x^{\prime}, y^{\prime}\right)\right\|\right\}\left\|c-c^{\prime}\right\| .
$$

Using $\|x\|,\left\|x^{\prime}\right\| \leq K$ and $\|y\|,\left\|y^{\prime}\right\| \leq L_{1}\left(\left\|b^{\prime}\right\|+\left\|A_{x}\right\| K\right)$, we obtain

$$
\left|v\left(b^{\prime}, c\right)-v\left(b^{\prime}, c^{\prime}\right)\right| \leq\left(K+L_{1}\left(\left\|b^{\prime}\right\|+\left\|A_{x}\right\| K\right)\right)\left\|c-c^{\prime}\right\| .
$$

The second part of the proof of Proposition 1 relies on the property that the distance of the optimal set of the linear relaxation of (5) to optimal points of (5) can be bounded with a constant that is independent of the choice of $b$ and $c$ (see [5, Theorem 1.2] or [6, Theorem 1]).

Acknowledgements This work was supported by the DFG Research Center MATHEON in Berlin and the BMBF under the grant 03SF0312E. We thank an anonymous referee whose report helped to improve an earlier version of this paper.

\section{References}

1. B. Bank, J. Guddat, D. Klatte, B. Kummer, and K. Tammer: Non-Linear Parametric Optimization, Akademie-Verlag, Berlin 1982.

2. B. Bank and R. Mandel: Parametric integer optimization, Akademie Verlag, Berlin 1988.

3. P. Billingsley: Convergence of Probability Measures, Wiley, New York 1968.

4. C. E. Blair and R. G. Jeroslow: The value function of a mixed integer program I, Discrete Mathematics 19 (1977), 121-138.

5. C. E. Blair and R. G. Jeroslow: The value function of a mixed integer program II, Discrete Mathematics 25 (1979), 7-19. 
6. W. Cook, A.M.H. Gerards, A. Schrijver, and É. Tardos: Sensitivity theorems in integer linear programming, Mathematical Programming 34 (1986), 251264.

7. A. Eichhorn and W. Römisch: Stochastic integer programming: limit theorems and confidence intervals, Mathematics of Operations Research 32 (2007), 118135 .

8. S. Engell, A. Märkert, G. Sand, and R. Schultz: Aggregated scheduling of a multiproduct batch plant by two-stage stochastic integer programming, $O p$ timization and Engineering 5 (2004), 335-359.

9. H. Heitsch and W. Römisch: A note on scenario reduction for two-stage stochastic programs, Operations Research Letters (2007) (to appear).

10. R. Henrion, C. Küchler, and W. Römisch: Discrepancy distances and scenario reduction in two-stage stochastic integer programming, Preprint, DFG Research Center Matheon "Mathematics for key technologies", 2007.

11. W. K. Klein Haneveld and M. H. van der Vlerk: Stochastic integer programming: General models and algorithms, Annals of Operations Research 85 (1999), 39-57.

12. F. Louveaux and R. Schultz: Stochastic integer programming, in Stochastic Programming, Handbooks in Operations Research and Management Science Vol. 10, Elsevier, Amsterdam, 2003, 213-266.

13. M. P. Nowak, R. Schultz, and M. Westphalen: A stochastic integer programming model for incorporating day-ahead trading of electricity into hydrothermal unit commitment, Optimization and Engineering 6 (2005), 163-176.

14. R. Nürnberg and W. Römisch: A two-stage planning model for power scheduling in a hydro-thermal system under uncertainty, Optimization and Engineering 3 (2002), 355-378.

15. S. T. Rachev: Probability Metrics and the Stability of Stochastic Models, Wiley, Chichester 1991.

16. S. T. Rachev and W. Römisch: Quantitative stability in stochastic programming: The method of probability metrics, Mathematics of Operations Research 27 (2002), 792-818.

17. R. T. Rockafellar and R. J-B Wets: Variational Analysis, Springer, Berlin 1998.

18. W. Römisch: Stability of stochastic programming problems, in Stochastic Programming, Handbooks in Operations Research and Management Science Vol. 10, Elsevier, Amsterdam, 2003, 483-554.

19. R. Schultz: On structure and stability in stochastic programs with random technology matrix and complete integer recourse, Mathematical Programming 70 (1995), 73-89.

20. R. Schultz: Rates of convergence in stochastic programs with complete integer recourse, SIAM Journal on Optimization 6 (1996), 1138-1152.

21. R. Schultz: Stochastic programming with integer variables, Mathematical Programming 97 (2003), 285-309.

22. S. Sen: Algorithms for stochastic mixed-integer programming models, Chapter 9 in Discrete Optimization (K. Aardal, G. L. Nemhauser, R. Weissmantel eds.), Handbooks in Operations Research and Management Science Vol. 12, Elsevier, Amsterdam 2005, 515-558.

23. D. Walkup and R. J-B Wets: Lifting projections of convex polyhedra, Pacific Journal of Mathematics 28 (1969), 465-475. 